\title{
Assessment of the relationship between serum squamous cell carcinoma antigen (SCC-Ag) concentration in patients with locally advanced squamous cell carcinoma of the uterine cervix and the risk of relapse
}

\author{
Sylwester Kubik ${ }^{1}$, Malgorzata Moszynska-Zielinska², Jacek Fijuth ${ }^{2,3}$, Adam Tomalczyk ${ }^{4}$, \\ Dorota Jesionek-Kupnicka ${ }^{5}$, Lidia Ura ${ }^{6}$, Leszek Marcin Gottwald ${ }^{2,3}$ \\ 1Department of Perinatology and Gynecology, Polish Mother's Memorial Hospital Research Institute of Lodz, Poland \\ ${ }^{2}$ Department of Teleradiotherapy, Regional Cancer Center, Copernicus Memorial Hospital of Lodz, Poland \\ ${ }^{3}$ Department of Radiotherapy, Chair of Oncology, Medical University of Lodz, Poland \\ ${ }^{4}$ Department of Nuclear Medicine, Regional Cancer Center, Copernicus Memorial Hospital of Lodz, Poland \\ ${ }^{5}$ Department of Pathology, Chair of Oncology, Medical University of Lodz, Poland \\ ${ }^{6}$ Department of Infections Control, Mazowiecki Memorial Hospital of Radom, Poland
}

\begin{abstract}
Introduction: Parameters that will help to identify patients with better and worse prognosis are sought in subjects with locally advanced squamous cell cervical carcinoma.

Aim of the study: To assess the relationship between squamous cell carcinoma antigen (SCC-Ag) concentration and the risk of relapse in patients with squamous cell cervical carcinoma staged IIB-IIIB.

Material and methods: The study group consisted of 52 patients with cervical squamous cell carcinoma staged II B $(n=39)$ and IIIB $(n=13)$. Serum SCC-Ag concentration was assessed prior to radiochemotherapy or radiotherapy and four weeks after treatment.

Results: The follow-up after treatment ranged from 1 to 33 months $(16.2 \pm 10.5)$. During follow-up, nine relapses were diagnosed (17.3\%). The concentration of SCC-Ag before the treatment was elevated in 41 cases $(78.8 \%)$ and in 11 cases $(21.2 \%)$ it was $\leq 2 \mathrm{ng} / \mathrm{ml}$. In all the patients with relapses SCC-Ag concentration before the treatment was elevated. Relapses were diagnosed in five patients with elevated SCC-Ag concentration after the treatment (55.6\%) and in four patients with normal SCC-Ag concentration after the treatment (9.3\%). There was a positive correlation between SCC-Ag concentration before and after the treatment and relapse occurrence.

Conclusions: Evaluation of serum SCC-Ag concentration in patients with locally advanced squamous cell cervical carcinoma before treatment is a valuable supplementary diagnostic tool and patients with high SCC-Ag concentration are at an increased risk of relapse. Due to the relationship between elevated serum SCC-Ag concentration after treatment and increased risk of relapse, these patients may require a more intensive post-treatment follow-up.
\end{abstract}

Key words: cervical carcinoma, squamous cell carcinoma antigen, chemoradiotherapy, relapse.

\section{Introduction}

Cervical carcinoma is the third most common malignant neoplasm of the genital organs in women in Poland, after endometrial carcinoma and ovarian carcinoma [1]. Squamous cell carcinoma accounts for about $80 \%$ of primary cervical malignancies $[2,3]$.

The results of treatment in locally advanced cervical carcinoma, i.e. stages IIB-IIIB according to International Federation of Gynecology and Obstetrics (FIGO) [2], depend on a number of factors related to the biology of the tumour and the general condition of patients. Staging, histology and grading are among the parameters characterizing the tumour evaluated in clinical practice. The duration of treatment, number of chemotherapy courses and haemoglobin concentration are also considered as prognostically important $[2,3]$. Despite continuous improvement of radiotherapy techniques and progress in systemic treatment, the results of treatment in these patients are still unsatisfactory [3]. Other parameters which will help to identify patients with better and worse prognosis are sought. 
The aim of this study was to assess the relationship between the concentration of squamous cell carcinoma antigen (SCC-Ag) and the risk of relapse in patients with locally advanced squamous cell cervical carcinoma.

Table 1. Clinical data of studied population

\begin{tabular}{lcccccc}
\hline Parameters & \multicolumn{2}{c}{$\begin{array}{l}\text { Non-relapsed } \\
\text { patients }\end{array}$} & \multicolumn{2}{l}{$\begin{array}{l}\text { Relapsed } \\
\text { patients }\end{array}$} & \multicolumn{2}{l}{ Total } \\
\cline { 2 - 7 } & $n$ & $\%$ & $n$ & $\%$ & $n$ & $\%$ \\
\hline $\begin{array}{l}\text { Number } \\
\text { of patients }\end{array}$ & 43 & 82.7 & 9 & 17.3 & 52 & 100 \\
\hline \begin{tabular}{l} 
Menopause \\
\hline before
\end{tabular} & 8 & 15.4 & 2 & 3.8 & 10 & 19.2 \\
\hline after & 35 & 67.3 & 7 & 13.5 & 42 & 80.8 \\
\hline Childbirth & & & & & & \\
\hline yes & 40 & 76.9 & 7 & 13.5 & 47 & 90.4 \\
\hline no & 3 & 5.8 & 2 & 3.8 & 5 & 9.6 \\
\hline Staging (FIGO) & & & & & & \\
\hline IIB & 36 & 69.2 & 3 & 5.8 & 39 & 75.0 \\
\hline IIIB & 7 & 13.5 & 6 & 11.5 & 13 & 25.0 \\
\hline Histology & & & & & & \\
\hline G1 & 7 & 13.5 & 0 & 0 & 7 & 13.5 \\
\hline G2 & 25 & 48.0 & 3 & 5.8 & 28 & 53.8 \\
\hline G3 & 11 & 21.2 & 6 & 11.5 & 17 & 32.7 \\
\hline Treatment & & & & & & \\
\hline RT-CHT & 38 & 73.1 & 9 & 17.3 & 47 & 90.4 \\
\hline RT & 5 & 9.6 & 0 & 0 & 5 & 9.6 \\
\hline $\begin{array}{l}\text { Median age } \\
\text { (years) }\end{array}$ & $60.0 \pm 10.2$ & $57.4 \pm 13.2$ & $59.5 \pm 10.9$ \\
\hline $\begin{array}{l}\text { Follow-up } \\
\text { (months) }\end{array}$ & $16.1 \pm 10.4$ & $17.7 \pm 9.3$ & $16.3 \pm 10.5$ \\
\hline & & & & & & \\
\hline
\end{tabular}
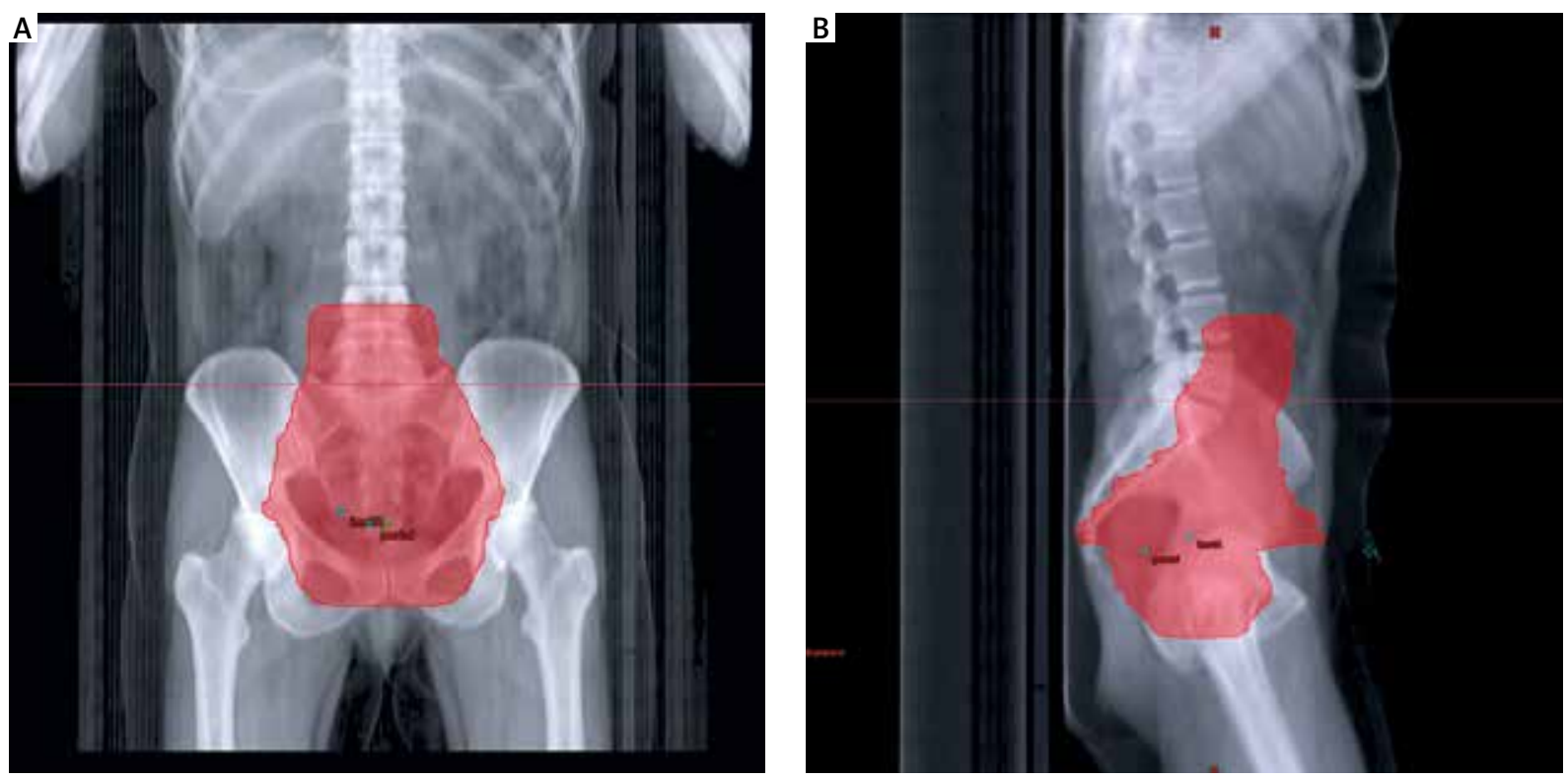

\section{Material and methods}

The study group consisted of 52 patients with cervical squamous cell carcinoma staged IIB $(n=39)$ and IIIB $(n=13)$ treated at the Department of Teleradiotherapy, Copernicus Memorial Hospital of Lodz in 2015-2017. Detailed clinical data of the studied population are presented in Table 1. Staging was evaluated clinically based on a pelvic magnetic resonance imaging (MRI) scan. The treatment scheme involved teleradiotherapy to the pelvis, uterus, both adnexa and regional lymph nodes (Fig. 1A, B) up to a dose of 44 Gy fractionated at 2 Gy with weekly injections of cisplatin at a dose of $40 \mathrm{mg} / \mathrm{m}^{2}$. In patients with contraindications to chemotherapy only teleradiotherapy was applied. After teleradiotherapy with or without chemotherapy was completed, highdose-rate (HDR) brachytherapy was implemented, fractionated at 7 Gy weekly for four weeks up to a total dose of $28 \mathrm{~Gy}$, or teleradiotherapy was continued up to a total were carried out in the oncological outpatient clinic. Serum SCC-Ag concentration was assessed prior to radiotherapy and four weeks after the end of treatment.

The SCC-Ag serum concentration standard for the laboratory where all measurements were performed was $0-2.0 \mathrm{ng} / \mathrm{ml}$. The study was approved by the Bioethics Commission of the Medical University of Lodz no. RN/1/1/15/KE. All data were analysed using Statistica 9.0 software (StatSoft, Tulsa, OK, USA). The $p$-value lower than 0.05 was considered as significant.

\section{Results}

When being diagnosed with primary cancer, the patients were aged between 29 and 78 years (median dose of 60 Gy. Follow-up appointments after treatment

Fig. 1. A, B) Irradiation field in teleradiotherapy of cervical carcinoma 
$59.5 \pm 10.9) ; 10$ patients (19.2\%) were before and 42 patients $(80.8 \%)$ after the menopause. Forty-seven patients $(90.4 \%)$ had a history of childbirth. Seven patients (13.5\%) had G1 tumours, 28 patients (53.8\%) G2 tumours and 17 patients (32.7\%) G3 cervical carcinomas. In 47 patients radiochemotherapy was applied and 5 patients received teleradiotherapy up to 44 Gy only. In 50 patients after teleradiotherapy, HDR brachytherapy was used and in 2 patients teleradiotherapy was continued up to a total dose of $60 \mathrm{~Gy}$. The follow-up care after treatment lasted from 1 month to 33 months (median $16.24 \pm 10.5$ ). During follow-up, 9 relapses (17.3\%) were found, including 4 local relapses and 5 distant relapses in the form of metastases to the lungs $(n=2)$, retroperitoneal lymph nodes $(n=2)$ and bones $(n=1)$.

Serum SCC-Ag concentration before the treatment was elevated in 41 cases $(78.8 \%)$ and in 11 cases $(21.2 \%)$ it was $\leq 2 \mathrm{ng} / \mathrm{ml}(p=0.003)$. At stage IIB it was $0.6-41.3 \mathrm{ng} / \mathrm{ml}$ (median $10.2 \pm 9.8$ ), and at stage IIIB it was 0.8-89.4 ng/ml (median $36.9 \pm 11.0 ; p<0.001$ ).

In all the patients with relapses serum SCC-Ag concentration before the treatment was elevated and ranged between 4.3 and $89.4 \mathrm{ng} / \mathrm{ml}$ (median $23.4 \pm 14.5$ ), and in 5 of the patients the level was above $10 \mathrm{ng} / \mathrm{ml}$. In 32 patients without relapses $(74.4 \%)$ the serum SCC$\mathrm{Ag}$ concentration before treatment was elevated, being above $10 \mathrm{ng} / \mathrm{ml}$ in 14 cases, whereas in 11 patients (25.6\%) it was within normal limits. Serum SCC-Ag concentration before treatment in the non-relapse group was lower than in the relapse group and ranged between 0.7 and $43.8 \mathrm{ng} / \mathrm{ml}$ (median $10.0 \pm 9.1 ; p<0.001$ ). In total, among 41 patients with elevated SCC-Ag levels before treatment, 9 (21.9\%) were diagnosed with a relapse of cervical carcinoma. None of the patients with a normal SCC-Ag concentration before treatment were diagnosed with a relapse. There was a positive correlation between SCC-Ag concentration before treatment and relapse $(r=0.40 ; p<0.05)$. There was a negative correlation between SCC-Ag concentration before treatment and time to relapse $(r=-0.55 ; p<0.05)$.

The frequency of elevated SCC-Ag concentration after treatment was lower than before treatment $(p<0.001$; Fig. 2). In all the relapse cases during the follow-up, the concentration of SCC-Ag assessed four weeks after the treatment decreased and ranged between 0.5 and $25.9 \mathrm{ng} / \mathrm{ml}$ (median $6.0 \pm 5.2$ ). In 4 of these cases the concentration of SCC-Ag after the treatment was $\leq 2.0 \mathrm{ng} / \mathrm{ml}$ (1 local relapse, 3 distant relapses). In 37 non-relapse cases the concentration of SCC-Ag after the treatment decreased, but it remained above the norm in 4 patients. In the other 6 cases the concentration of SCC-Ag both before and after the treatment was $\leq 2 \mathrm{ng} / \mathrm{ml}$. The concentration of SCC-Ag after the treatment in the non-relapse group was lower than in the relapse group and ranged between 0.4 and $9.2 \mathrm{ng} / \mathrm{ml}$ (median $1.2 \pm 0.9 ; p<0.001$ ).
A total of 5 out of 9 patients (55.6\%) with elevated SCC-Ag concentration after the treatment and 4 out of 43 patients $(9.3 \%)$ with normal SCC-Ag concentration after the treatment were diagnosed with recurrent cervical carcinoma $(p<0.001)$. There was a positive correlation between SCC-Ag concentration four weeks after the end of treatment and the occurrence of relapses ( $r=0.33$; $p<0.05)$. There was a negative correlation between SCC-Agconcentration after treatment and time to relapse $(r=-0.31 ; p<0.05)$.

\section{Discussion}

This study is valuable because it is prospective, all the SCC-Ag measurements were performed at the same laboratory at predetermined time points related to the treatment, and all the patients were treated according to the same protocol by one medical team. There are no studies in patients with locally advanced squamous cell cervical carcinoma that in prospective observation analyse SCC-Ag concentrations at two time points, i.e. before and after treatment.

Although the relationship between serum levels of SCC-Ag and parameters of cervical carcinoma patients has been the subject of numerous publications, this problem is still unclear. According to Gadducci et al. the concentration of SCC-Ag assessed before treatment is elevated in $28-88 \%$ of patients with squamous cell cervical carcinoma and there is a correlation between it and staging, tumour size, depth of stromal infiltration, invasion of lymphatic space and lymph nodes [4]. Similar observations were described by Liu et al. after the analysis of 308 cases in stages IB-IIA, which confirmed that in cases with deep cervical infiltration the concentration of SCC-Ag is elevated [5]. Gaarenstroom, on the other hand, follow-

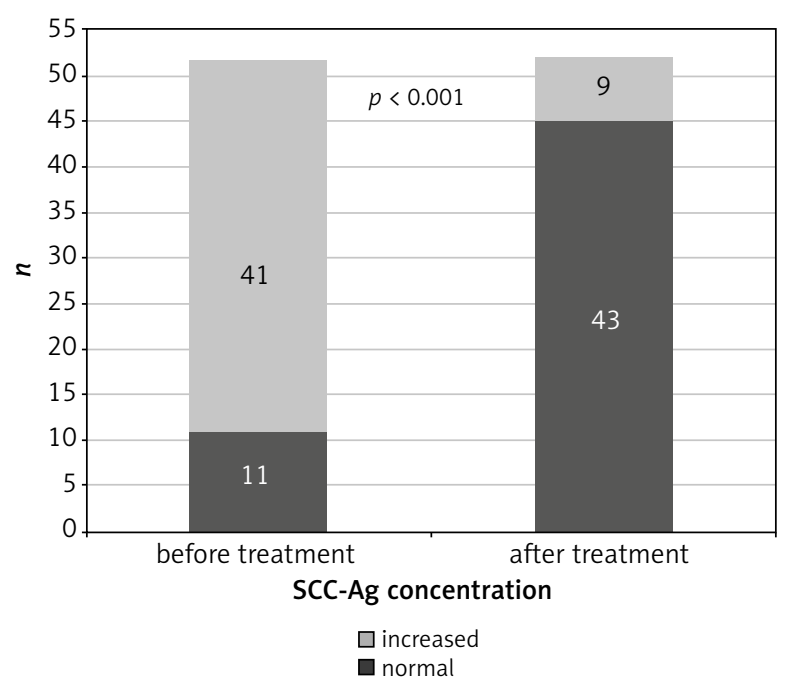

Fig. 2. Plasma SCC-Ag concentration in patients with locally advanced squamous cell cervical carcinoma before and after treatment 
ing an analysis of 78 patients, pointed out the relation between elevated SCC-Ag concentration and the parametrial infiltration and the presence of metastases in the lymphatic system [6]. However, not all authors agree with these observations. Salvatici et al., having analysed 197 cases of squamous cell cervical carcinoma in stages I-II, did not confirm the relationship between the concentration of SCC-Ag, staging and grading of squamous cell cervical carcinoma [7]. Bonfrer et al. also reported a lack of correlation between the concentration of SCC-Ag before treatment and staging of cervical carcinoma [8]. Our results in a group of 52 patients with locally advanced squamous cell cervical carcinoma indicate that the serum SCC-Ag concentration at the time of diagnosis is higher in most of these patients and at stage IIIB reaches higher values than in IIB.

Another issue is the possibility of using SCC-Ag concentration assessment in diagnostics of metastases to the lymphatic system. Liu et al. established that the concentration of SCC-Ag is elevated in patients with metastases of cervical carcinoma to the lymphatic system [5]. Analogical views are also presented by other authors [4, 9]. Based on a meta-analysis of 17 studies covering 3985 patients with squamous cell cervical carcinoma, Zhou et al. found that the concentration of SCC-Ag correlates with the presence of metastases to the retroperitoneal lymph nodes and this assay can be used commonly with imaging methods to diagnose metastases to the lymphatic system [9].

The relationship between serum SCC-Ag concentration and cervical carcinoma relapse has also been the subject of numerous studies. In the analysis by Esajas, among 225 cases of early cervical squamous cervical carcinoma, relapses were diagnosed in 35 cases, whereas in 26 cases (74.3\%) they were accompanied by elevated levels of SCC-Ag [10]. Similar observations have also been described by other authors [6-8]. Charakorn et al. analysed 61 publications devoted to the relation between SCC-Ag concentration and cervical carcinoma relapse and found that the relative risk of relapse at a high vs. low concentration of SCC-Ag before treatment is 2.44 , and when it is assessed after treatment it increases up to 3.91 [11]. In our study, no relapse of cervical carcinoma was observed in any of the patients whose SCC-Ag concentration had not been elevated before the treatment, and the elevated marker concentration four weeks after the end of treatment was associated with an increased risk of relapse. It is believed that if the concentration of SCC-Ag decreases after treatment and then increases again during observation, in $46-92 \%$ of cases such an occurrence precedes the clinical diagnosis of a relapse, usually by two to eight months [4]. So far, the relationship between the site of the relapse and the concentration of SCC-Ag has not been confirmed [7].

An increased concentration of SCC-Ag is also associated with an increased risk of death due to cancer.
However, it is a less important factor than staging and tumour size $[6,8]$. In the study by Esajas, the average survival period after the diagnosis of a relapse was shorter in cases of relapses with a higher SCC-Ag concentration than in cases of relapses without any elevated SCC-Ag concentration (9 vs. 20 months) [10]. We did not study this relationship since the post-treatment observation period was not long enough.

\section{Conclusions}

Evaluation of serum SCC-Ag concentration in patients with locally advanced squamous cell cervical carcinoma before treatment is a valuable supplementary diagnostic tool, and patients with high SCC-Ag concentration are at increased risk of relapse.

Due to the relationship between elevated serum SCC-Ag concentration after treatment and increased risk of relapse, these patients may require a more intensive post-treatment follow-up.

\section{Disclosure}

The authors report no conflict of interest.

\section{References}

1. The National Cancer Registry. Malignant neoplasms in Poland in 2015. http://www.onkologia.org.pl

2. Jach R, Sznurkowski J, Bidziński M, et al. Recommendations of the Polish Gynecological Society for the diagnosis and treatment of cervical cancer. Curr Gynecol Oncol 2017; 15: 24-33.

3. Marth C, Landoni F, Mahner S, et al. Cervical cancer: ESMO Clinical Practice Guidelines for diagnosis, treatment and follow-up. Ann Oncol 2017; 28 (Suppl. 4): 72-83.

4. Gadducci A, Tana R, Cosio S, et al. The serum assay of tumour markers in the prognostic evaluation, treatment monitoring and follow-up of patients with cervical cancer: a review of the literature. Crit Rev Oncol Hematol 2008; 66: 10-20

5. Liu CZ, Zeng HX, Zhao JJ, et al. The validity of using human squamous cell carcinoma associated antigen and cytokeratin 19 fragment antigen 21-1 to predict postoperative adjuvant radiotherapy for nonbulky early-stage squamous cell carcinoma of the cervix. Int J Gynecol Cancer 2017; 27: 994-1000.

6. Gaarenstroom KN, Bonfrer JM, Kenter GG, et al. Clinical value of pretreatment serum Cyfra 21-1, tissue polypeptide antigen, and squamous cell carcinoma antigen levels in patients with cervical cancer. Cancer 1995; 76: 807-813.

7. Salvatici M, Achilarre MT, Sandri MT, et al. Squamous cell carcinoma antigen (SCC-Ag) during follow-up of cervical cancer patients: Role in the early diagnosis of recurrence. Gynecol Oncol 2016; 142: 115-119.

8. Bonfrer JM, Gaarenstroom KN, Korse CM, et al. Cyfra 21-1 in monitoring cervical cancer: a comparison with tissue polypeptide antigen and squamous cell carcinoma antigen. Anticancer Res 1997; 17: 2329-2334.

9. Zhou Z, Li W, Zhang F, et al. The value of squamous cell carcinoma antigen (SCCa) to determine the lymph nodal metastasis in cervical cancer: A metaanalysis and literature review. PLoS One 2017; 12: e0186165.

10. Esajas MD, Duk JM, de Bruijn HW, Aalders JG, et al. Clinical value of routine serum squamous cell carcinoma antigen in follow-up of patients with earlystage cervical cancer. J Clin Oncol 2001; 19: 3960-3966.

11. Charakorn C, Thadanipon K, Chaijindaratana S, et al. The association between serum squamous cell carcinoma antigen and recurrence and survival of patients with cervical squamous cell carcinoma: A systematic review and meta-analysis. Gynecol Oncol 2018; 150: 190-200. 